\title{
THE CRITICAL EARTHQUAKE MODEL: OBSERVATIONS IN CRITICAL AREAS OF THE BROADER AEGEAN AREA
}

\author{
Kourouzidis, M. C. ${ }^{1}$, Karakaisis, G. F. ${ }^{2}$, Papazachos, B. C. ${ }^{2}$, and Makropoulos, C. ${ }^{3}$ \\ ${ }^{1}$ Institute of Geodynamics, National Observatory of Athens, PO Box 20048, GR-11810 Athens, \\ Greece,m.kour@gein.noa.gr \\ ${ }^{2}$ Geophysical Laboratory, School of Geology, Aristotle University of Thessaloniki, PO Box 352-1, \\ 54124, Thessaloniki, karakais@geo.auth.gr, basil@lemnos.geo.auth.gr \\ ${ }^{3}$ Department of Geophysics and Geothermy, Faculty of Geology, National \& Kapodistrian \\ University of Athens, Univ. Campus, Ilisia, GR-15784, Athens, Greece, kmacrop@uoa.gr
}

\begin{abstract}
The time variation of the cumulative Benioff strain, $S$, in several critical regions of past mainshocks which occurred in Greece and surrounding area has been examined, in an attempt to check the validity of the critical point concept. For this reason, data concerning the critical regions of twelve strong mainshocks, which have recently been defined by other scientists, have been used and intermediate-sized earthquakes which occurred before and after each mainshock were collected. It was found that the cumulative seismic crustal deformation released by these intermediate magnitude events was accelerating before all the mainshocks considered, whereas the deformation release rate during the respective postshock periods was, in general, lower than the preshock ones.
\end{abstract}

\section{INTRODUCTION}

Observations on large scale space-time clustering of earthquakes have attracted the interest of seismologists long ago (Willis 1924, Imamura 1937, Gutenberg \& Richter 1954, Tocher 1959, among others). These observations reported changes in the occurrence rate of intermediate-sized earthquakes prior to large mainshocks in California and Japan and later resulted in the definition of the seismic cycle (Fedotov 1968, Mogi 1969).

Many scientists applied methods of Statistical Physics in an attempt to model the large earthquake generation process (Rundle 1989, Smalley et al. 1985). However, the study of this process within the framework of the chaos theory during 1980's led to the suggestion that the earthquake generation process can be considered as a critical phenomenon that culminates in a critical point, that is, the mainshock (Sornette \& Sornette 1990, Allègre \& Le Mouel 1994, among others). The crust of the earth can be considered as a system, which is always in a dynamic state: stresses act within the crust and cause deformation, faults slip with various velocities, etc. These physical processes form a complex system, which exhibits non-linear behavior and the earthquake generation is one of these processes (Keilis-Borok et al. 2001).

Among the main issues on which research on seismicity patterns has been focused during the last decade or so, is whether the crust is in a continuous state of self-organized criticality (Bak et al. 1988, Bak \& Tang 1989) or whether it repeatedly approaches and retreats from a critical state (Sammis \& Smith 1999). Within this context, studies on the behavior of the intermediate magnitude seismic activity prior to large earthquakes mainly in California resulted in the identification of accelerating seismicity, expressed in terms of seismic moment, energy or Benioff strain release before these earthquakes, which follows a power law (Varnes 1989, Sykes \& Jaumé 1990, Bufe \& Varnes 1993, Jaumé \& Sykes 1999). That is:

$$
S(t)=A+B\left(t_{c}-t\right)^{m}
$$

where $S(t)$ is the cumulative Benioff strain (square root of seismic energy) of all earthquakes occurred at time $t$ and are termed preshocks, $t_{c}$ is the mainshock origin time, and $A, B, m$ are parameters which are calculated by the available data (Bufe \& Varnes 1993). 
The scientific hypothesis that a mainshock in a certain area can be considered as the culmination (critical point) of a dynamical process (approach to the critical state) which takes place within this area, has to be supported by observations showing that significant changes of the properties of the area (for example seismicity rate, etc.) take place in this area before and after the mainshock generation. Papazachos (2003) examining the time variation of the cumulative Benioff strain released by intermediate-sized events before and after the 28 March 1970 large $(M=7.0)$ mainshock in NW Turkey, reported that accelerating strain release rate was observed in a broad area (critical area) prior to the mainshock, which was followed by its aftershocks, that is, shocks that occurred in the mainshock rupture zone. Then, the postshock strain release rate within the critical area varied linearly with time.

In the present work the results of the examination of the properties of the large scale, spacetime clustering of intermediate magnitude earthquakes before strong mainshocks, which occurred in the broader Aegean area, are presented (Kourouzidis 2003), with the aim being to find additional evidence in favor of the critical earthquake model.

\section{METHOD AND DATA}

The proper definition of the critical area, that is, the area in which the deformation released by intermediate magnitude events accelerates with time and culminates in the generation of a mainshock, was first proposed by Bowman et al. (1998) on the basis of the curvature parameter $\mathrm{C}$. This parameter is defined as the ratio of the root mean square error of the power-law fit (relation 1) to the corresponding linear fit error and practically quantifies the degree of deviation of the released Benioff strain from linearity (acceleration); small C values $(\leq 0.7$, Bowman et al. 1998 ) ensure that the accelerating seismicity law (1) describes the data much more adequately than the standard linear time variation. Very recently, Papazachos \& Papazachos $(2000,2001)$ and Papazachos et al. (2003) proposed additional constraints, which facilitate the definition of a critical region of a past or an impending mainshock. These constraints are expressed by several relations between the parameters involved and relate: (a) the radius, $\mathrm{R}$ (in $\mathrm{km}$ ), of the circle with area equal to the area of the elliptical region with the mainshock magnitude, $\mathrm{M}$, and the mean deformation rate in the area, $s_{r}$ (in Joule $e^{1 / 2} / \mathrm{yr}$ and per $10^{4} \mathrm{~km}^{2}$ ), (b) the duration of the preshock sequence, $t_{p}$ (in yrs), with $s_{r_{1}}$ (c) the mean rate of deformation during the accelerating deformation period, $A / t_{p}$, with the long term rate of seismic deformation, $S_{r}$, and (d) the mainshock magnitude, $M$, with the average magnitude of the three largest preshocks, $\mathrm{M}_{13}$ (relations 2-5).

$$
\begin{array}{ll}
\log R=0.42 M-0.30 \log s_{r}+1.28 & \sigma=0.08 \\
\log t_{p}=3.87-0.45 \log s_{r} & \sigma=0.09 \\
\log \left(A / t_{p}\right)=1.01 \log S_{r} & \sigma=0.04 \\
M=M_{13}+0.60 & \sigma=0.10
\end{array}
$$

In order to quantify the compatibility of these relations with observations, a parameter $\mathrm{P}$ was defined (Papazachos \& Papazachos 2001), which is the average value of the probabilities that each of these four parameters $\left(\mathrm{R}, t_{p}, A, \mathrm{M}\right)$, attains a value close to its expected one, using a Gaussian probability density function based on the deviations reported in equations (2), (3), (4) and (5). Furthermore, Papazachos et al. (2002a) have defined a quality measure, q, given by the relation:

$$
q=\frac{P}{C m}
$$

in an attempt to simultaneously evaluate: a) the compatibility of an accelerating seismic deformation with the behavior of past real preshock sequences (large $P$ ), b) the deviation of the variation with time of the seismic deformation from linearity (small C) and c) the degree of seismic acceleration (small $\mathrm{m}$ ). Investigation of preshock sequences of strong mainshocks $(M \geq 6.4)$, which occurred in the Aegean since 1950, has led to the adoption of the following cut-off values:

$$
\mathrm{C} \leq 0.60, \mathrm{~m} \leq 0.35, \mathrm{P} \geq 0.45, \mathrm{q} \geq 3.0
$$

Papazachos et al. (2002b) studying the dynamic process in the Aegean lithosphere that results in the generation of a strong earthquake, defined the critical regions of 18 mainshocks which occurred in the Aegean and surrounding area during 1950-2000. They also examined the possibility of retrospective prediction of these mainshocks. From this data set 12 mainshocks were selected, for which the duration of the postshock period was equal to the duration of the preshock period. The 
basic focal parameters of these mainshocks along with the characteristics of their respective elliptical critical regions are listed in Table 1.

Table 1. The basic focal parameters of the shallow mainshocks that occurred in Greece and surrounding areas along with information on their respective critical regions (Papazachos et al. 2002b). For each mainshock the code number, the occurrence date, the epicenter coordinates ( $\mathrm{N}$ latitude, $\mathrm{E}$ longitude), the center of the corresponding elliptical critical region and its magnitude, $\mathrm{M}$, are given in the first five columns. In the next three columns the length of the major semi-axis of the ellipse, a $(\mathrm{km})$, its azimuth, $z$, and the ellipticity, e, are listed. The three remaining columns give information on the data considered, that is, the minimum magnitude, $M_{\min }$, of the intermediate-sized events and the years delimiting the period examined.

\begin{tabular}{ccccccccccc}
\hline No & Date & epicenter & ell. center & $\mathbf{M}$ & $\mathbf{a}(\mathbf{k m})$ & $\mathbf{z}$ & $\mathbf{e}$ & $\mathbf{M}_{\min }$ & $\mathbf{t}_{\mathbf{s}}$ & $\mathbf{t}_{\mathbf{e}}$ \\
\hline 1 & 17.12 .1952 & 34.424 .5 & 34.924 .4 & 7.0 & 274 & 30 & 0.90 & 5.2 & 1922 & 1983 \\
2 & 18.03 .1953 & 40.027 .5 & 39.427 .3 & 7.4 & 469 & 0 & 0.95 & 5.6 & 1920 & 1983 \\
3 & 12.08 .1953 & 38.120 .6 & 37.920 .6 & 7.2 & 385 & 90 & 0.95 & 5.3 & 1930 & 1976 \\
4 & 30.04 .1954 & 39.122 .3 & 39.122 .3 & 7.0 & 313 & 90 & 0.95 & 5.3 & 1932 & 1976 \\
5 & 16.07 .1955 & 37.627 .1 & 38.026 .1 & 7.0 & 313 & 150 & 0.95 & 5.2 & 1920 & 1990 \\
6 & 09.07 .1956 & 36.626 .0 & 36.425 .8 & 7.5 & 348 & 150 & 0.70 & 5.6 & 1929 & 1983 \\
7 & 25.04 .1957 & 36.528 .6 & 36.128 .0 & 7.2 & 388 & 150 & 0.95 & 5.4 & 1930 & 1984 \\
8 & 19.02 .1968 & 39.525 .0 & 39.625 .2 & 7.1 & 313 & 30 & 0.95 & 5.3 & 1933 & 2001 \\
9 & 09.07 .1980 & 39.322 .8 & 39.322 .8 & 6.5 & 91 & 0 & 0.70 & 4.5 & 1965 & 1995 \\
10 & 24.02 .1981 & 38.222 .9 & 38.023 .2 & 6.7 & 333 & 0 & 0.95 & 5.2 & 1966 & 1996 \\
11 & 17.01 .1983 & 38.120 .2 & 37.220 .8 & 7.0 & 385 & 60 & 0.95 & 5.3 & 1967 & 1999 \\
12 & 05.07 .1983 & 40.327 .2 & 40.027 .8 & 6.4 & 130 & 60 & 0.90 & 4.6 & 1970 & 1996 \\
\hline
\end{tabular}

\section{RESULTS}

In figures 1-4 the elliptical critical regions for the 12 mainshocks listed in table 1 are shown. Black and grey circles in the elliptical critical region of each map correspond to the epicenters of the preshocks and postshocks of the respective mainshock (large black circle), whereas the large grey diamond denotes the center of this region. For each critical region, all earthquakes with magnitudes $M \geq M_{\min }$ which occurred during the time period defined by $t_{s}$ and $t_{e}$ were collected and the cumulative Benioff strain, $S$, was calculated by the relation:

$$
S(t)=\sum_{i=1}^{n(t)}\left[E_{i}(t)\right]^{1 / 2}
$$

where $E$ is the seismic energy released by the events within the critical region (in Joules) and can be calculated by the formula logE=1.5M+4.7 (Papazachos \& Papazachos 2000). Next to each map a plot of the time variation of the Benioff strain, $\mathrm{S}(\mathrm{t})$, released by the earthquakes that occurred in the respective critical region on the left is shown. Black and gray circles indicate the deformation released during the preshock and the postshock period, respectively. The large diamond corresponds to the deformation released by the mainshock whereas small diamonds show the deformation released by its aftershocks (Kourouzidis 2003).

It can be observed that in all cases the strain release rate during the preshock time period is higher than the release rate during the postshock period. This behavior is more prominent in those cases in which the duration of the preshock period and consequently the postshock one, is relatively small. For example, from the data listed in table 1 it comes out that the duration of the preshock time period in the cases $9-12$ was about 13 to 16 years, while in all the other cases the preshock sequence was between 22 and 33 years. It has also to be noted that the size of the critical areas of the Magnesia 1980 and NW Turkey 1983 mainshocks (earthquakes 9 and 12 in table 1) is smaller than the size of the other critical areas.

In the cases 1-8 of those listed in table 1 and shown in the respective figures, the Benioff strain release rate during the postshock time period, despite the fact that it is lower in general than the 

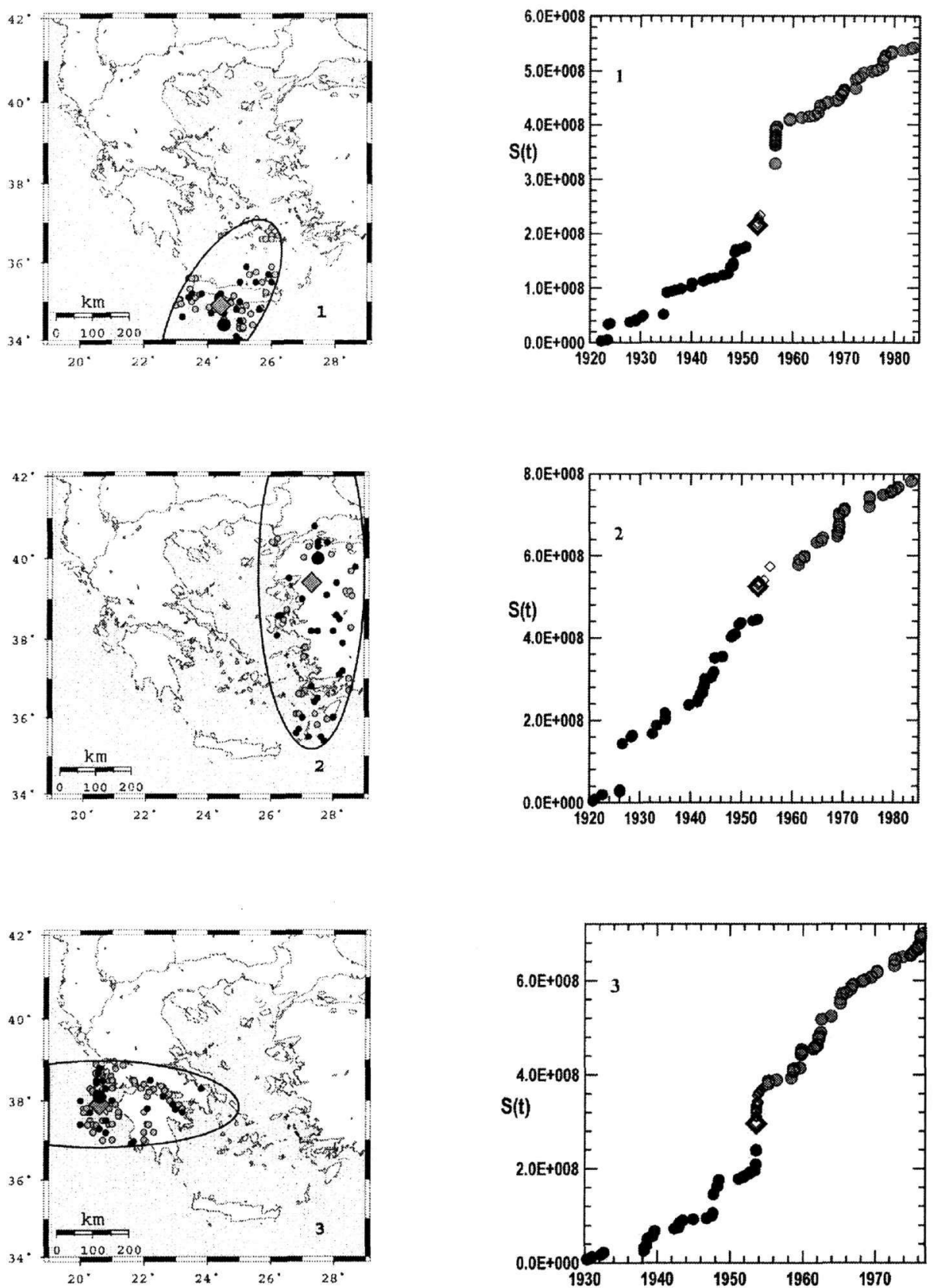

Figure 1. (Left) The critical areas of the earthquakes with code numbers $1,2,3$ of table 1 . Black and gray circles correspond to the epicenters of the earthquakes that occurred in the critical areas before and after the corresponding mainshocks. The large black circles and the diamonds denote the mainshock epicenters and the centers of the critical regions, respectively. (Right) The time variations of the cumulative Benioff strain, $S(t)$, released by all $M \geq M_{\text {min }}$ shocks which occurred in the critical regions during the examined time periods. Black and gray circles indicate the deformation released during the preshock and the postshock periods, respectively. The large diamonds correspond to the deformation released by the mainshocks whereas small diamonds show the deformation released by its aftershocks (Kourouzidis 2003). 

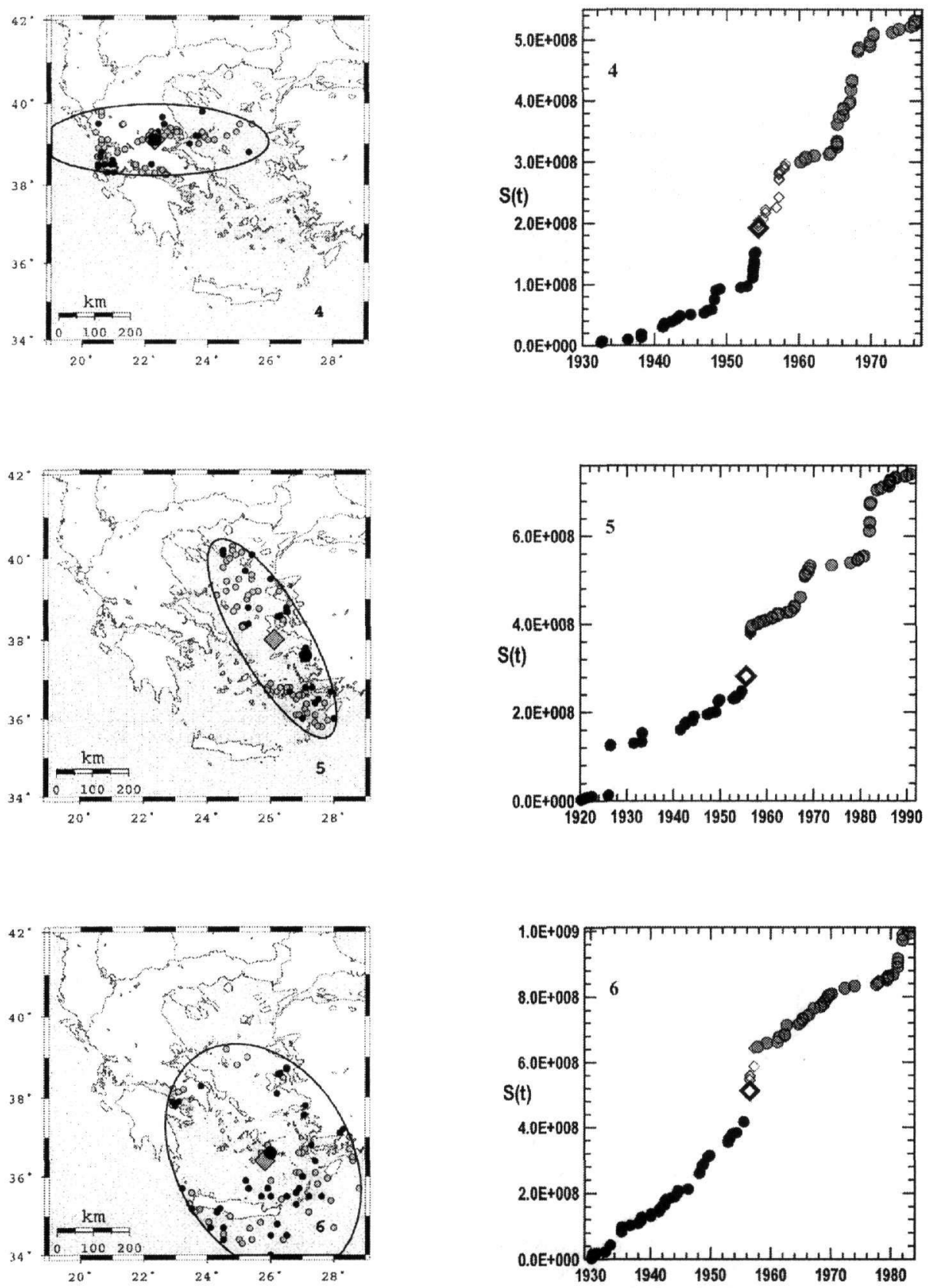

Figure 2. The critical areas of the earthquakes with code numbers $4,5,6$ of table 1 (left) and the corresponding time variations of the cumulative Benioff strain, $S(t)$, released by all $M \geq M_{\min }$ shocks which occurred in the critical areas during the examined time periods (right) (Kourouzidis 2003). Explanation as in figure 1. 

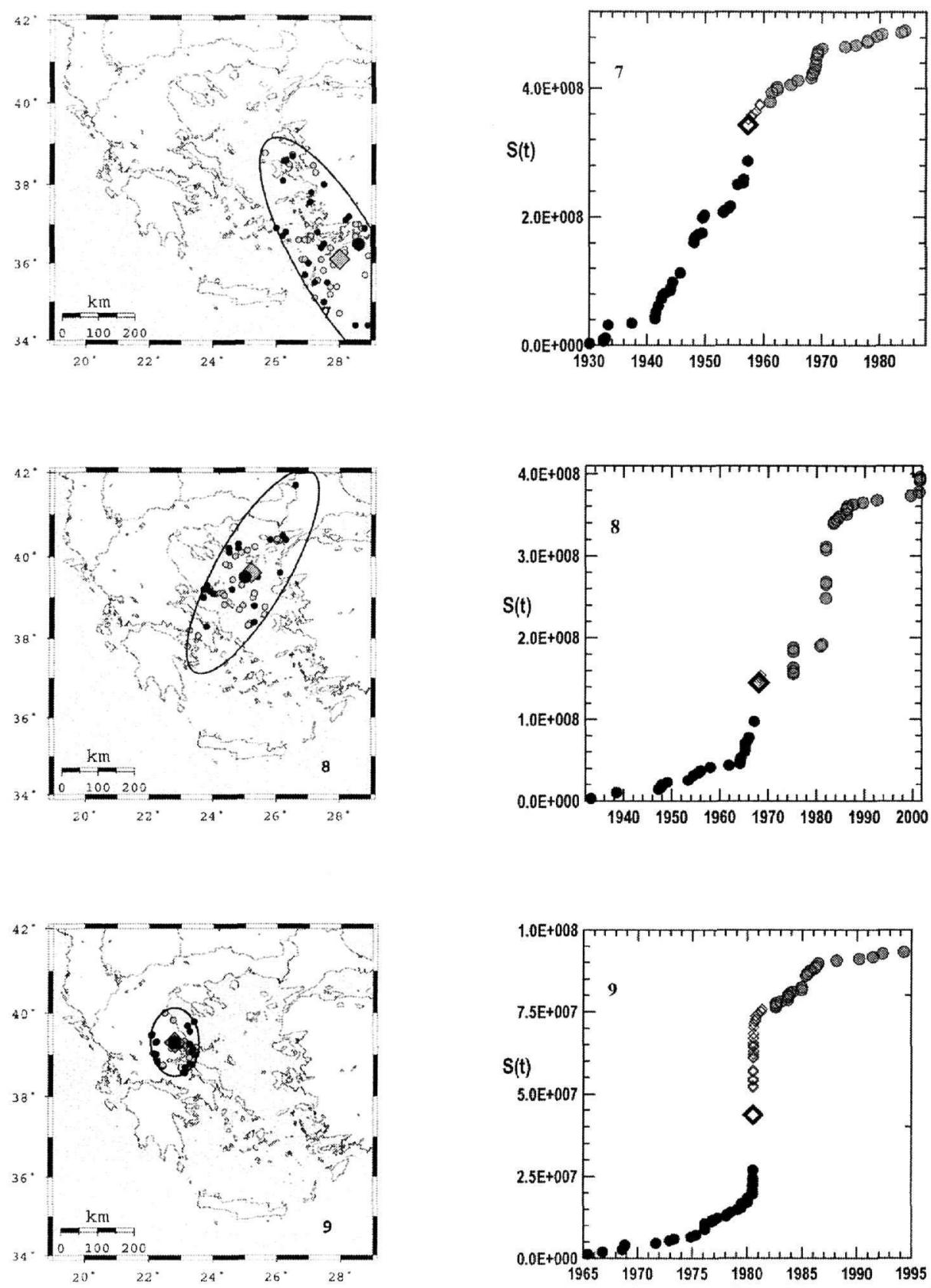

Figure 3. The critical areas of the earthquakes with code numbers $7,8,9$ of table 1 (left) and the corresponding time variations of the cumulative Benioff strain, $S(t)$, released by all $M \geq M_{\min }$ shocks which occurred in the critical areas during the examined time periods (right) (Kourouzidis 2003). Explanation as in figure 1. 

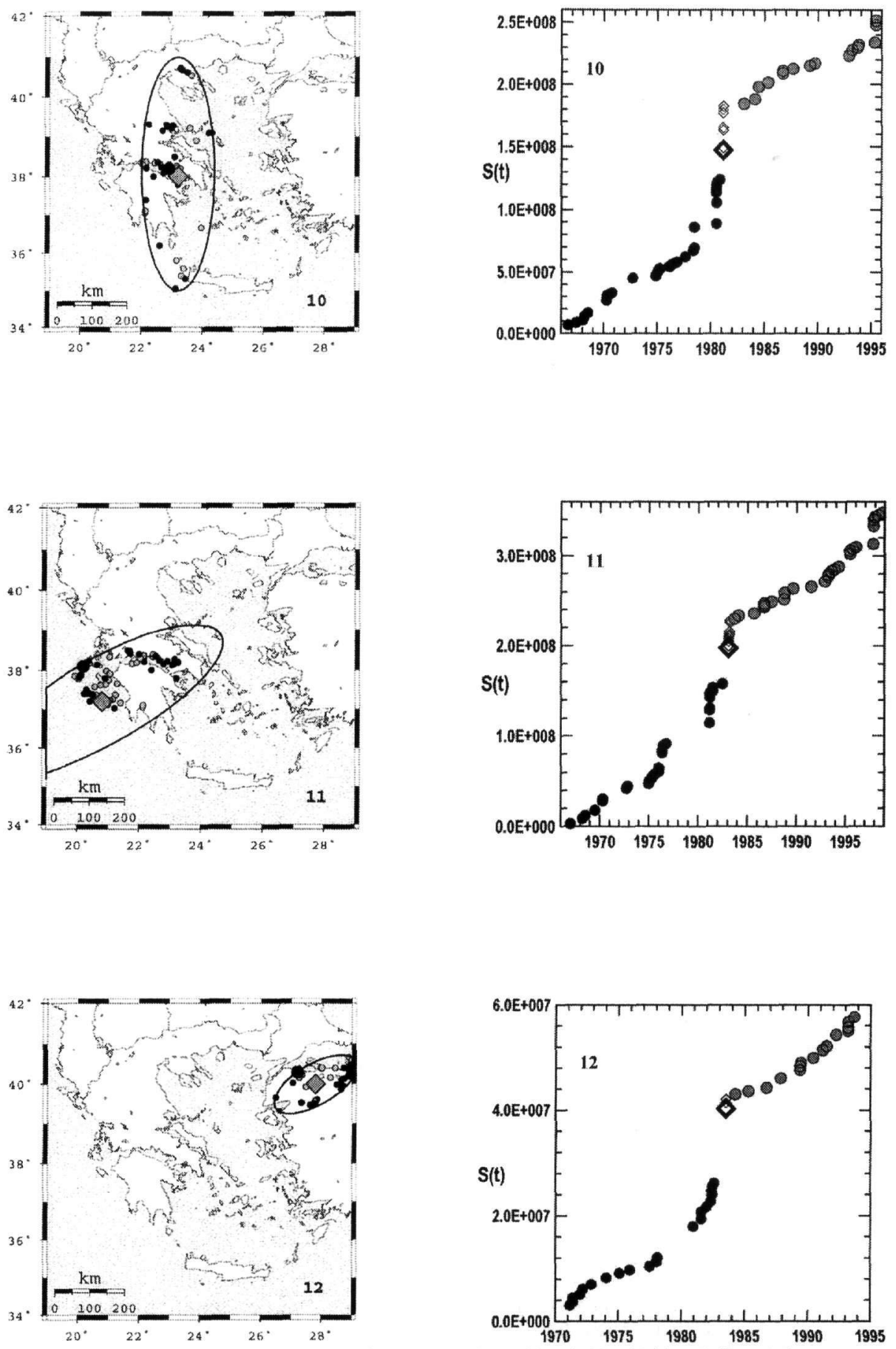

Figure 4. The critical areas of the earthquakes with code numbers 10,11,12 of table 1 (left) and the corresponding time variations of the cumulative Benioff strain, $S(t)$, released by all $M \geq M_{\min }$ shocks which occurred in the critical areas during the examined time periods (right) (Kourouzidis 2003). Explanation as in figure 1. 
rate of the preshock period, exhibits transient excitations as, for example, in the case (4) (Central Greece, 1954, M=7.0). It can be seen in figure 2 that the strain release rate during the postseismic period increased abruptly in 1967 when the March 4, $1967(M=6.6)$ earthquake occurred in Northern Aegean and probably part of the critical region of this mainshock coincides with part of the critical area of the 1954 mainshock.

Another observation concerns preshocks which belong to different critical regions (i.e. cases 3$4,2-5,5-6$, etc.). A tentative explanation may be that we can not rule out the possibility that several earthquakes contribute to the approach to criticality (to a mainshock generation) in different regions simultaneously. Furthermore, it has to be noted that a mainshock and its postshock activity in a certain region may trigger another mainshock in an adjacent region, as it has been shown in studies of static stress changes (Stein, 1999; Papadimitriou and Sykes, 2001). More work is needed, however, to clarify this issue.

\section{DISCUSSION AND CONCLUSIONS}

A central issue for the earthquake generation process within the concept of self-organized criticality is whether the crust continuously maintains itself in a critical state, as originally proposed by Bak \& Tang (1989) or it can repeatedly approach and retreat from a critical state. The explicit consequence of the former view is that all small earthquakes will have the same probability of growing into a large event, hence "..earthquakes are inherently unpredictable" (Geller et al. 1997). In the last decade, however, there is a growing number of observations, supported by results obtained through models of simple cellular automata with loss and/or structural complexity, that a large earthquake in a region perturbs it away from the critical state and that methods of statistical physics can be used to monitor the return of the region toward criticality and the next large earthquake (Triep and Sykes, 1997; Sammis and Smith, 1999). There exists a considerable number of studies which have shown that large earthquakes tend to be preceded by clusters of intermediate-sized events; Sykes \& Jaumé (1990) examining the seismicity in the San Francisco Bay Area, have found that large earthquakes are preceded by a cluster of intermediate-sized events (within two magnitude units of the main shock) in a large surrounding region. Furthermore, Knopoff et al. (1996) showed that all 11 earthquakes in California since 1941 with magnitudes greater than 6.8 were preceded by an increase in the rate of occurrence of earthquakes with magnitudes greater than 5.1. Jaumé \& Sykes (1999) summarize a large amount of relevant observations and cite earlier references.

The above mentioned studies indicate that a key question to the mainshock generation process is whether the accelerating deformation that leads to the critical point (mainshock) ends by the mainshock or continues after its occurrence. We believe that seismological data can be used to resolve this ambiguity. The results presented in this paper favor the former view, since in all cases studied the deformation rate in the postshock period is lower than the rate in the preshock period; the accelerating strain release rate observed in the preshock time period probably is a manifestation of the growing correlation length within the critical area that has been considered as an indicator for critical point behavior prior to large earthquakes (Zöller et al. 2001). The generation of the mainshock destroys the criticality in this area in which a period of seismic quiescence follows. Then, this process is repeated and the area approaches criticality again as long-range stress correlation develops.

\section{AGKNOWLEDGEMENTS}

We are grateful to the two anonymous reviewers whose comments helped us to clarify certain issues. The maps were made by the use of the GMT software (Wessell and Smith, 1995). This work has been partially supported by the Greek Planning and Protection Organization (OASP), (Res. Comm. AUTH project 20242).

\section{REFERENCES}

Allègre C.J. \& Le Mouel J.L. 1994. Introduction of scaling techniques in brittle failure of rocks. Phys. Earth Planet. Inter., 87, 85-93. 
Bak. P., Tang C. \& Wiesenfeld K. 1988. Self-organized criticality. Phys. Rev. A, 38, 364-374.

Bak P. \& Tang C. 1989. Earthquakes as a self-organized critical phenomenon. J. geophys. Res., 94, 1563515637.

Bowman D.D., Ouillon G., Sammis C.G., Sornette A. \& Sornette D. 1998. An observational test of the critical earthquake concept. J. geophys. Res., 103, 24359-24372.

Bufe C.G. \& Varnes D.J. 1993. Predictive modelling of seismic cycle of the Great San Francisco Bay Region. J. geophys. Res., 98, 9871-9883.

Fedotov S. A. 1968. The seismic cycle, quantitative seismic zoning and long-term seismic forecasting, in Seismic Zoning of the USSR, pp. 133-166, ed. Medvedev, S., Moscow.

Geller R.J., Jackson D.D., Kagan, Y.Y. \& Mulargia, F. 1997. Earthquakes cannot be predicted. Science, 275, 1616-1617

Gutenberg B. \& Richter C.F. 1954. Seismicity of the Earth and Associated phenomena. Hafner, New York.

Imamura A. 1937. Theoretical and applied seismology. Maruzen, Tokyo.

Jaumé S.C. \& Sykes L.R. 1999. Evolving towards a critical point: A review of accelerating seismic moment/energy release rate prior to large and great earthquakes. Pure appl. Geophys., 155, 279-306.

Keilis-Borok V., Ismail-Zadeh A., Kossobokov V. \& Shebalin P. 2001. Non-linear dynamics of the lithosphere and intermediate-term earthquake prediction. Tectonophysics, 338, 247-260.

Knopoff L., Levshina T., Keilis-Borok V.J. \& Mattoni C. 1996. Increased long-range intermediate-magnitude earthquake activity prior to strong earthquakes in California. J. geophys. Res. 101, 5779-5796.

Kourouzidis M.C. 2003. Study of seismic sequences in Greece and its contribution to earthquake prediction. Ph. D. Thesis, Univ. of Thessaloniki, pp. 150 (in Greek with English abstract).

Mogi K. 1969. Some features of the recent seismic activity in and near Japan. 2, activity before and after great earthquakes. Bull. Earthquake Res. Inst., Univ. of Tokyo, 47, 395-417.

Papadimitriou, E.E. \& Sykes, L.R., 2001. Evolution of the stress field in the Northern Aegean Sea (Greece). Geophys. J. Int., 146, 747-759.

Papazachos B.C. 2003. Chaos in seismology and earthquake prediction. International Workshop on Galaxies and Chaos, Theory and Observations. Athens, Greece, 16-19 September 2002, 1-22.

Papazachos B.C. \& Papazachos C.B. 2000. Accelerated preshock deformation of broad regions in the Aegean area. Pure appl. Geophys., 157, 1663-1681.

Papazachos C.B. \& Papazachos B.C. 2001. Precursory accelerating Benioff strain in the Aegean area, Ann. Geofis., 144, 461-474.

Papazachos C.B., Karakaisis G.F., Savaidis A.S. \& Papazachos B.C., 2002a. Accelerating seismic crustal deformation in the southern Aegean area. Bull. Seismol. Soc. Am., 92, 570-580.

Papazachos C.B., Karakaisis G.F. \& Scordilis, E.M. 2002b. Results of a retrospective prediction of past strong mainshocks in the broader Aegean area by application of the accelerating seismic deformation method. Proc. XXVIII ESC General Assembly, Genoa, 1-6 September, 14pp.

Papazachos C.B., Karakaisis G.F., Scordilis E.M. \& Papazachos B.C., 2003. Global observational properties of the critical earthquake model. (submitted to Bull. Seism. Soc. Am.).

Rundle J.B. 1989. A physical model for earthquakes. J. geophys. Res., 94, 2839-2855.

Sammis C. G. \& Smith S. W. 1999. Seismic cycles and the evolution of stress correlation in cellular automaton models of finite fault networks. Pure appl. Geophys., 155, 307-334.

Smalley R.F., Turcotte D.L. \& Solla S.A. 1985. A renormalization-group approach to the stick-slip behavior of faults. J. geophys. Res. 90, 1894-1900.

Sornette A. \& Sornette D. 1990. Earthquake rupture as a critical point. Consequences for telluric precursors. Tectonophysics, 179, 327-334.

Stein, R.S., 1999. The role of stress transfer in earthquake occurrence. Nature, 402, 605-609.

Sykes L.R. \& Jaumé S. 1990. Seismic activity on neighboring faults as a long term precursor to large earthquakes in the San Francisco Bay area. Nature, 348, 595-599.

Tocher D. 1959. Seismic history of the San Francisco bay region. Calif. Div. Mines Spec. Rep., 57, 39-48.

Triep E. G. \& Sykes L. R. 1997. Frequency of occurrence of moderate to great earthquakes in intracontinental regions: implications for changes in stress, earthquake prediction, and hazards assessments. J. geophys. Res., 102, 9923-9948.

Varnes D. J. 1989. Predicting earthquakes by analyzing accelerating precursory seismic activity. Pure appl. Geophys., 130, 661-686.

Wessel P. \& Smith W. 1995. New version of the Generic Mapping Tools. EOS Trans. Amer. Geophys. U., 76: 329.

Willis B. 1924. Earthquake risk in California and earthquake districts. Bull. Seism. Soc. Am., 14, 9-25.

Zöller G., Hainzl S. \& Kurths J. 2001. Observation of growing correlation length as an indicator for critical point behavior prior to large earthquakes. J. geophys. Res., 106, 2167-2175. 